\title{
Holographic superfluid solitons with backreaction
}

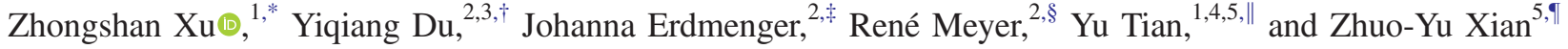 \\ ${ }^{1}$ School of Physical Sciences, University of Chinese Academy of Sciences, Beijing 100049, China \\ ${ }^{2}$ Institute for Theoretical Physics and Astrophysics and Würzburg-Dresden Cluster of Excellence ct.qmat, \\ Am Hubland 97074 Würzburg, Germany \\ ${ }^{3}$ Department of Physics, Hanyang University, 222 Wangshimni-ro, Sungdong-gu, \\ Seoul 04763, South Korea \\ ${ }^{4}$ Center for Theoretical Physics, Massachusetts Institute of Technology, \\ Cambridge, Massachusetts 02139, USA \\ ${ }^{5}$ Institute of Theoretical Physics, Chinese Academy of Sciences, Beijing 100190, China
}

(Received 4 January 2020; accepted 24 March 2020; published 9 April 2020)

\begin{abstract}
Solitons are important nonperturbative excitations in superfluids. For holographic superfluids, we numerically construct "dark" solitons that have the symmetry-restored phase at their core. A central point is that we include the gravitational backreaction of the matter fields, which becomes important at low temperatures. We study in detail the properties of these solitons under variation of the backreaction strength via tuning the gravitational constant. In particular, the depletion fraction of the particle number density at the core of the solitons is carefully investigated. In agreement with the probe-limit analysis, the depletion fraction shows the same qualitative behavior as in Bogoliubov-de Gennes theory, even if the backreaction is included. We find that the depletion decreases with increasing backreaction strength. Moreover, the inclusion of backreaction enables us to obtain the effective energy density of solitons within holography, which together with an evaluation of the surface tension leads to a simple physical explanation for the snake instability of dark solitons.
\end{abstract}

DOI: 10.1103/PhysRevD.101.086011

\section{INTRODUCTION}

Gauge/gravity duality [1-3] is a powerful tool to describe strongly coupled and correlated systems. Many problems associated with strongly interacting condensed matter physics are tractable in this setup [4]. One of these problems is unconventional superfluidity $[5,6]$.

Superfluidity is a collective quantum phenomenon occurring in both bosonic and fermionic systems at low temperatures. In particular, fermionic systems can interpolate in a smooth way between the formation and condensation of loosely bound Cooper pairs (BCS superconductivity), and Bose-Einstein condensation (BEC) of preformed bosonic

\footnotetext{
*xuzhongshan16@mails.ucas.edu.cn

†du@physik.uni-wuerzburg.de

Corresponding author.

erdmenger@physik.uni-wuerzburg.de

${ }^{\S}$ rene.meyer@physik.uni-wuerzburg.de

"Corresponding author.

ytian@ucas.ac.cn

"xianzy@itp.ac.cn

Published by the American Physical Society under the terms of the Creative Commons Attribution 4.0 International license. Further distribution of this work must maintain attribution to the author(s) and the published article's title, journal citation, and DOI. Funded by SCOAP ${ }^{3}$.
}

molecules. This is known as the BCS-BEC crossover (see Ref. [7] for a review), which has been realized by cooling fermionic gases to ultra-low temperatures and tuning the interactions between the fermions with a controllable external magnetic field in the laboratory. The qualitative essence of this crossover can be understood from the phase diagram depicted in Fig. 1: the horizontal axis interpolates between the BCS regime of a weakly attractive interaction between fermions and the BEC regime of very strong attraction. Above the pairing onset temperature $T^{*}$, the system is a normal Fermi liquid consisting of the unpaired fermions on the BCS side. On the BEC side, the strong attractive interaction binds the fermions together to form bosonic molecules, which at large enough temperatures form a Bose liquid. As the temperature on the BCS side decreases, loosely bound Cooper pairs of fermions start to form at $T<T^{*}$ and condense below a critical temperature $T_{c}$. On the BEC side, Bose-Einstein condensation of the bosonic molecules occurs for $T<T_{c}$. Between these two regimes and at $T<T_{c}$, there exists a strongly coupled regime of unconventional superfluidity around the point of infinite scattering length $\frac{1}{k_{F} a}=0$, which is known as the unitary Fermi gas.

Superfluids are quantum fluids which can sustain nonperturbative excitations such as solitons and vortices $[8,9]$. 


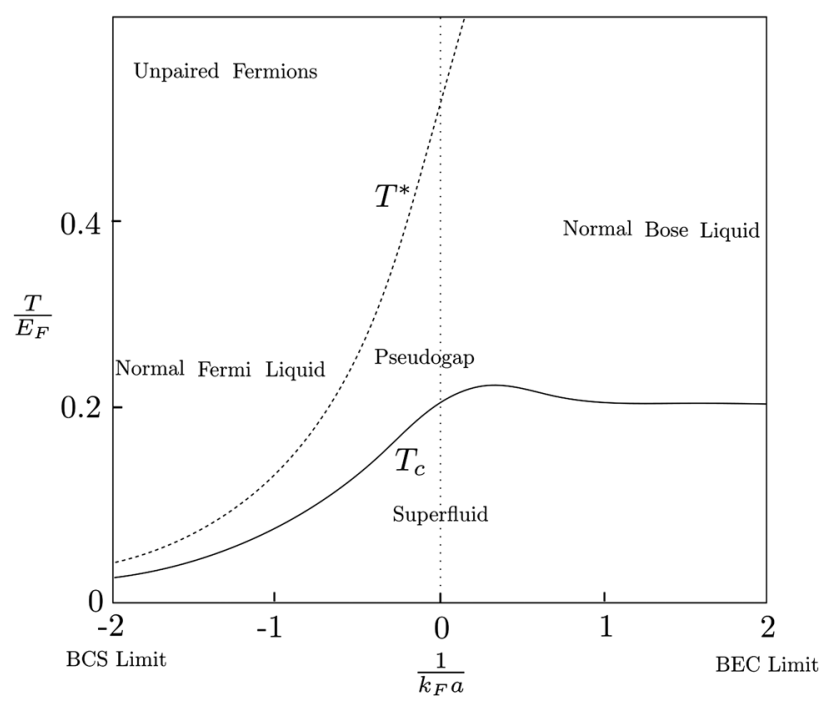

FIG. 1. Qualitative phase diagram reproduced from Ref. [7] of the BCS-BEC crossover as a function of temperature $T / E_{F}$ and coupling $1 / k_{F} a$, where $k_{F}$ is the Fermi momentum and $a$ is the scattering length. $T^{*}$ is the pairing onset temperature, while $T_{c}$ is the critical temperature for the superfluidity.

One kind of soliton is the so-called dark soliton, which interpolates between the two phases of the system. ${ }^{1}$ The core of dark solitons is in the normal state where the condensate vanishes, while asymptotically far away from the core the soliton is in the superfluid state. The core properties can be used to provide insights into the microscopic structure of the superfluid [11]. It was found that, at zero temperature, solitons display distinctively different core properties in the BEC and BCS regimes. For the BEC superfluid, solitons have vanishing core particle number density, because bosonic molecules fully condense below the critical temperature, and there are no noncondensed states available. But in the core of the BCS dark soliton, there are still unpaired fermionic atoms or loosely bound Cooper pairs in excited states, which lead to a nonvanishing core particle number density. At finite temperature, even in the BEC superfluid there will be a small number of uncondensed particles arising from thermal excitation [12]. In order to quantify these properties, the depletion fraction of the particle number density at the core is defined as $\frac{\rho_{\max }-\rho_{\min }}{\rho_{\max }}$. Here, $\rho_{\min }$ is the density at the core, while $\rho_{\max }$ is

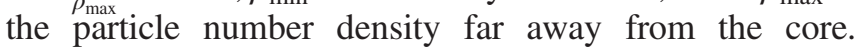
The depletion fraction was investigated in Bogoliubovde Gennes theory in Ref. [11], where it was found that in the BEC regime the core contains almost no particles, i.e., the depletion is almost $100 \%$, while in the BCS regime the soliton core still contains some particles at very low temperatures, with a depletion of about $30 \%$. Furthermore,

\footnotetext{
${ }^{1}$ Dark solitons include gray solitons with definite velocity and black solitons that are static [10]. We focus on black solitons in this work.
}

during the crossover from the $\mathrm{BCS}$ regime to the $\mathrm{BEC}$ regime, the depletion correspondingly undergoes a continuous variation from $30 \%$ to $100 \%$.

Holographic methods can be used to describe unconventional superconductivity and superfluidity $[5,6]$. Holographic dark solitons in the probe limit, i.e., neglecting the backreaction on the black-brane space-times forming the background, have been obtained in Refs. $[8,12,13]$. The depletion fraction for the particle number density was calculated in the two different quantizations possible in AdS/CFT, i.e., standard and alternative quantization. As explained in more detail below, both quantizations are related by a double-trace deformation. By comparing the holographic results with the depletion characteristics described above, a condensate with standard (alternative) quantization was found to show similar characteristics as in the BCS (BEC) regime in Ref. [12]. For ease of comparison, we will refer to the condition of standard (alternative) quantization as the BCS (BEC) case. Moreover, the authors of Refs. $[8,12]$ conjectured that one may implement the BCS-BEC crossover in holographic superfluid systems by varying the scaling dimension of the condensing operator.

It was also argued in Ref. [14] that by introducing double-trace deformations of the charged scalar, it is possible to model the interaction between fermionic constituents of the boundary system. By continuously tuning the coupling constant (denoted as $\kappa$ below) of the deformation, the salient features of the physics of the BCS-BEC crossover can be captured. In fact, the two independent arguments in Refs. [8,12,13] and Ref. [14] are related: according to Refs. [15-18], perturbing the large $\mathcal{N}$ boundary theory by a relevant double-trace deformation corresponds to imposing mixed boundary conditions $\psi_{+}=\kappa \psi_{-}$ for a scalar field in the bulk, where $\kappa$ is related to the coupling constant of the double-trace deformation. $\kappa=0$ $(\kappa=-\infty)$ is the boundary condition for alternative (standard) quantization. Adding the perturbation will trigger a renormalization group (RG) flow from the original conformal field theory (CFT) in the UV to another conformal fixed point in the IR. Under this flow, the conformal dimension of $\mathcal{O}$ varies between $\Delta_{-}$in the UV and $\Delta_{+}$in the IR, i.e., the two inequivalent boundary CFTs $\Delta=\Delta_{ \pm}$can be recovered as two limits of the same deformed theory.

The argument of Refs. [8,12,13] leaves an important caveat: while the experimental results are obtained at nearly zero temperature, the holographic probe limit that ignores the backreaction of the matter fields onto the metric is known to break down in the low-temperature regime. In particular, the condensate in the alternative quantization diverges near zero temperature [19], which is a sign of the backreaction becoming important at low temperatures. In this work, we hence study the behavior of dark solitons in a holographic superfluid system including backreaction. The dark soliton configurations are constructed by numerically solving the Einstein equations coupled to the matter fields. In particular, 
we employ the DeTurck method for finding stationary solutions, first introduced in AdS/CFT in Ref. [20] and further developed in Ref. [21]. This method can reformulate the Einstein equations into a manifestly elliptic form by adding a covariant gauge-fixing term to the Einstein equations, which gives a well-posed boundary value problem.

Another drawback of the probe limit is that the boundary stress-energy tensor of the condensate cannot be investigated, which conceals information about important thermodynamic quantities, such as the effective energy (mass) and entropy of the soliton. Taking into account the backreaction allows us to extract these quantities. Interestingly, it turns out that our result for the effective energy (mass) of the dark soliton, together with the surface tension of our holographic dark solitons, is consistent with previous expectations $[22,23]$ for the physical mechanism of a particular instability of dark solitons: the so-called snake instability. The snake instability is an instability of solitons under transverse perturbations, leading to the spontaneous formation of a snake-like bending of the solitons. The snake instability was observed in different physical systems [24-26], and has attracted much theoretical attention [23,27-30]. In holography, the authors of Ref. [31] identified the snake instability of holographic superfluids in the probe limit via the appearance of unstable quasinormal modes in the bulk, and observed the final decay of the "snake" into vortexantivortex pairs. The investigation of Ref. [31] was systematic but not as intuitive as effective arguments from mechanics or hydrodynamics (see, e.g., Refs. [22,23]). In this work we holographically confirm the explanation of the snake instability of dark solitons $[22,23]$ by calculating the negative effective mass responsible for the self-acceleration effect [22], as well as the positive surface tension responsible for the spontaneous generation of ripples on the soliton [23].

This paper is organized as follows. In the next section, we briefly introduce our holographic superfluid model and analyze the Ansätze and boundary conditions necessary for solving the equations of motion. In Sec. III, our numerical scheme and main numerical results are discussed. Section IV is devoted to the thermodynamics of the holographic dark soliton and to holographically confirming the mechanism of the snake instability. A summary and outlook are included in Sec. V.

\section{HOLOGRAPHIC SETUP}

We work with the simplest holographic superfluid model which requires gravity coupled to a Maxwell field $A_{\mu}$ and a massive charged scalar field $\Psi$ with charge $e$. The bulk action reads

$$
\begin{aligned}
S= & \int d^{4} x \sqrt{-g}\left[\frac{1}{2 \kappa_{4}^{2}}(R-2 \Lambda)\right. \\
& \left.-\frac{1}{e^{2}}\left(\frac{1}{4} F_{\mu \nu} F^{\mu \nu}+|D \Psi|^{2}+m^{2}|\Psi|^{2}\right)\right]
\end{aligned}
$$

where we have rescaled the gauge field $A_{\mu}$ and the scalar $\Psi$ to $\frac{A_{\mu}}{e}$ and $\frac{\Psi}{e}$ compared to the original form $[5,6] . L$ is the anti-de Sitter (AdS) radius related to the cosmological constant as $\Lambda=-\frac{3}{L^{2}}$, and $m$ is the mass of the charged scalar. The covariant derivative is $D_{\mu}=\nabla_{\mu}-i A_{\mu}$, where $\nabla_{\mu}$ is the Christoffel covariant derivative with respect to the background metric $g_{\mu \nu} . F_{\mu \nu}=\partial_{\mu} A_{\nu}-\partial_{\nu} A_{\mu}$ is the field strength. In the rest of the paper we set $L=1$. The equations of motion derived from the action take the following form:

$$
\begin{gathered}
R_{\mu \nu}-\Lambda g_{\mu \nu}-\frac{2 \kappa_{4}^{2}}{e^{2}}\left\{\frac{1}{2}\left[D_{\mu} \Psi\left(D_{\nu} \Psi\right)^{\dagger}+D_{\nu} \Psi\left(D_{\mu} \Psi\right)^{\dagger}+g_{\mu \nu} m^{2}|\Psi|^{2}\right]\right. \\
\left.+\left(\frac{1}{2} F_{\mu \sigma} F_{\nu}{ }^{\sigma}-\frac{g_{\mu \nu}}{8} F_{\rho \sigma} F^{\rho \sigma}\right)\right\}=0 \\
D^{\mu} D_{\mu} \Psi-m^{2} \Psi=0 \\
\nabla_{\mu} F^{\mu \nu}=i g^{\mu \nu}\left[\Psi^{\dagger}\left(D_{\mu} \Psi\right)-\Psi\left(D_{\mu} \Psi\right)^{\dagger}\right]
\end{gathered}
$$

In the probe limit $\frac{2 \kappa_{4}^{2}}{e^{2}} \ll 1$, the backreaction of the terms involving the gauge field and the charged scalar on the background geometry in Eq. (2) can be neglected. In this limit, one can first solve the Einstein equations $R_{\mu \nu}-\Lambda g_{\mu \nu}=0$ for the fixed background metric $g_{\mu \nu}$, and then solve the matter equations (3) and (4) on top of that fixed background. Once $\frac{2 \kappa_{4}^{2}}{e^{2}}$ is not small this is no longer possible, and the full coupled set of equations (2)-(4) have to be solved. In this work we are interested in the effect of backreaction. In the following we will set the charge of the scalar $e=1$ and define the backreaction parameter $\epsilon \equiv 2 \kappa_{4}^{2}$ as a measure of the strength of backreaction.

In the absence of the charged scalar in Eq. (1), the solution of the Einstein equations is the ReissnerNordström-AdS black brane

$$
\begin{gathered}
d s^{2}=\frac{1}{z^{2}}\left[-f(z) d t^{2}+\frac{d z^{2}}{f(z)}+d z^{2}+d y^{2}\right], \\
f(z)=1-\left(1+\frac{\epsilon \mu^{2} z_{+}^{2}}{4}\right)\left(\frac{z}{z_{+}}\right)^{3}+\frac{\epsilon \mu^{2} z_{+}^{2}}{4}\left(\frac{z}{z_{+}}\right)^{4}, \\
A=\mu\left[1-\left(\frac{z}{z_{+}}\right)\right] d t,
\end{gathered}
$$

where $\mu$ is the chemical potential and $z_{+}$parametrizes the black-brane temperature via

$$
T=\frac{1}{4 \pi z_{+}}\left(3-\frac{\epsilon \mu^{2} z_{+}^{2}}{4}\right) .
$$

For numerical convenience [32], we make the following radical coordinate transformation with $z_{h} \equiv \frac{1}{z_{+}}$: 


$$
z=\frac{1-r^{2}}{z_{h}}
$$

In order to construct the backreacted geometries, we use the following Ansatz compatible with staticity and translation invariance in the second boundary direction $y$ :

$$
\begin{gathered}
d s^{2}=\frac{z_{h}^{2}}{\left(1-r^{2}\right)^{2}}\left[-Q_{1} f(r) d t^{2}+\frac{4 r^{2} Q_{2} d r^{2}}{z_{h}^{2} f(r)}\right. \\
\left.+Q_{4}\left(d x-\frac{2 r}{z_{h}} Q_{3} d r\right)^{2}+Q_{5} d y^{2}\right], \\
\Psi=\left(\frac{1-r^{2}}{z_{h}}\right) Q_{6}, \\
A=\mu r^{2} Q_{7} d t .
\end{gathered}
$$

Here $\left\{Q_{i} \mid i=1,2, \ldots, 7\right\}$ are functions of $r$ and $x$ to be determined by solving Eqs. (2)-(4). In the coordinate (9), the conformal boundary is located at $r=1$, while the horizon is at $r=0$ where the regularity of $Q_{i}$ must be imposed. Expanding the equations of motion near the horizon as a power series in $r$ and requiring the leading order to vanish yields the boundary conditions

$\left.Q_{1}\right|_{r=0}=\left.Q_{2}\right|_{r=0} ;\left.\quad\left(\partial_{r} Q_{j}\right)\right|_{r=0}=0, \quad j=2,3, \ldots 7$.

In particular, the Dirichlet condition for $Q_{1}$ in Eq. (13) ensures that the temperature of the black brane is still given by Eq. (8). At the conformal boundary, we demand that the metric approaches $\mathrm{AdS}_{4}$, i.e.,

$\left.Q_{1}\right|_{r=1}=\left.Q_{2}\right|_{r=1}=\left.Q_{4}\right|_{r=1}=\left.Q_{5}\right|_{r=1}=1,\left.Q_{3}\right|_{r=1}=0$.

In the asymptotically AdS regime, the scalar field $\Psi$ behaves in the $z$ coordinate as

$$
\Psi=\psi_{-} z^{\Delta_{-}}+\psi_{+} z^{\Delta_{+}}+\ldots
$$

Here $\Delta_{ \pm}=3 / 2 \pm \sqrt{9 / 4+m^{2} L^{2}}$ is the conformal dimension of the operator $\mathcal{O}$ dual to $\psi$. We choose $m^{2} L^{2}=-2$. According to the AdS/CFT correspondence, in the standard quantization, one identifies $\psi_{-}$with the source of the operator $\mathcal{O}_{2}$ having the conformal dimension $\Delta_{+}=2$, while $\psi_{+}$is regarded as the response (expectation value). Alternatively, one can exchange the roles of $\psi_{-}$and $\psi_{+}$in the dual field theory, i.e., $\psi$ is dual to the operator $\mathcal{O}_{1}$ having the conformal dimension $\Delta_{-}=1$ with source $\psi_{+}$and expectation value $\psi_{-}$. Since we want to investigate a spontaneously generated condensate in the absence of sources, we will set $\psi_{-}=0$ (standard case) or $\psi_{+}=0$ (alternative case) on the conformal boundary. Generally, the mixed boundary condition $\psi_{+}=\kappa \psi_{-}$can also be imposed, which is known as the double-trace boundary condition since it corresponds to a deformation of the dual theory by adding a term $\sim \int d^{3} x \mathcal{O}^{\dagger} \mathcal{O}$ to its boundary action $S_{\text {bdry }}$ [15-17]. Finally, the gauge field admits the usual UV expansion

$$
A_{t}=\mu-\rho z+\ldots
$$

$\mu$ is interpreted as the chemical potential, and $\rho$ is the charge (or particle number) density. Since the ground state of the system is conformal, we can scale out one dimensionful quantity. Throughout this paper, we do so by normalizing all dimensionful quantities with the chemical potential, which we set to the fixed value $\mu=5.6 .^{2}$

\section{NUMERICAL SCHEME AND RESULTS}

We employ the DeTurck method to numerically solve the Einstein equations; for a recent review, see Ref. [33]. This method consists of adding the gauge-fixing term $\frac{1}{2} L_{\xi} g_{\mu \nu}=\nabla_{(\mu} \xi_{\nu)}$ to the Einstein equations (2), which breaks all diffeomorphisms and yields elliptic EinsteinDeTurck equations,

$$
\begin{gathered}
R_{\mu \nu}-\Lambda g_{\mu \nu}-\epsilon\left\{\frac{1}{2}\left[D_{\mu} \Psi\left(D_{\nu} \Psi\right)^{\dagger}+D_{\nu} \Psi\left(D_{\mu} \Psi\right)^{\dagger}+g_{\mu \nu} m^{2}|\Psi|^{2}\right]\right. \\
\left.+\left(\frac{1}{2} F_{\mu \sigma} F_{\nu}{ }^{\sigma}-\frac{g_{\mu \nu}}{8} F_{\rho \sigma} F^{\rho \sigma}\right)\right\}-\nabla_{(\mu} \xi_{\nu)}=0
\end{gathered}
$$

Here the DeTurck vector $\xi^{\nu} \equiv g^{\rho \sigma}\left[\Gamma_{\rho \sigma}^{\nu}(g)-\bar{\Gamma}_{\rho \sigma}^{\nu}(\bar{g})\right]$ is constructed from the difference of the Christoffel symbols of the metric $g_{\mu \nu}$ (which we aim to solve for) and a reference metric $\bar{g}_{\mu \nu}$. The reference metric has to have the same asymptotics and symmetries as the metric $g_{\mu \nu}$ we are trying to solve for. In our scheme, we take the standard AdS Reissner-Nordström metric (5), corresponding to $Q_{1}=Q_{2}=Q_{4}=Q_{5}=1$ and $Q_{3}=0$ in Eq. (10). We then find solutions to the Einstein-DeTurck equation with the constraint condition $\xi^{\mu}=0$, which ensures that our solution coincides with a solution to the Einstein equations (2). ${ }^{3}$

The nonlinear partial differential equations (PDEs) (3), (4), and (17), together with the boundary conditions (13)-(14) are then solved via the Newton-Kantorovich method. To be specific, we first linearize the PDEs and then discretize the linear partial differential equations into algebraic equations via the standard pseudospectral procedure, where we represent unknown functions as linear combinations of Chebyshev polynomials in the $z$ coordinate and a Fourier series in the $x$ coordinate. ${ }^{4}$ Our integration

\footnotetext{
${ }^{2}$ This value turns out to be numerically convenient in terms of the convergence speed of our code.

${ }^{3}$ We have checked that $\left|\xi^{2}\right|<10^{-10}$ when the size of the grids reaches $30 \times 150$.

${ }^{4}$ Since a single soliton has no periodicity in the $x$ direction, for the simplicity of the spatial boundary conditions and efficiency of the numerics we follow Ref. [34] and instead construct the double soliton (kink-antikink) configuration, and then analyze a single soliton.
} 


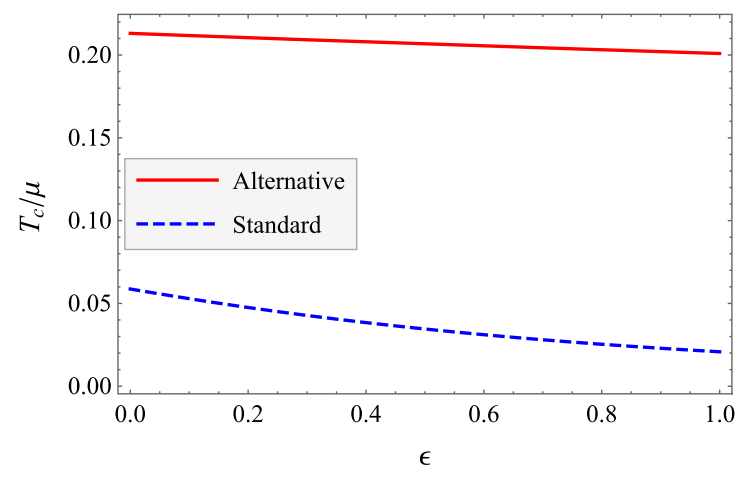

(a) Critical temperature

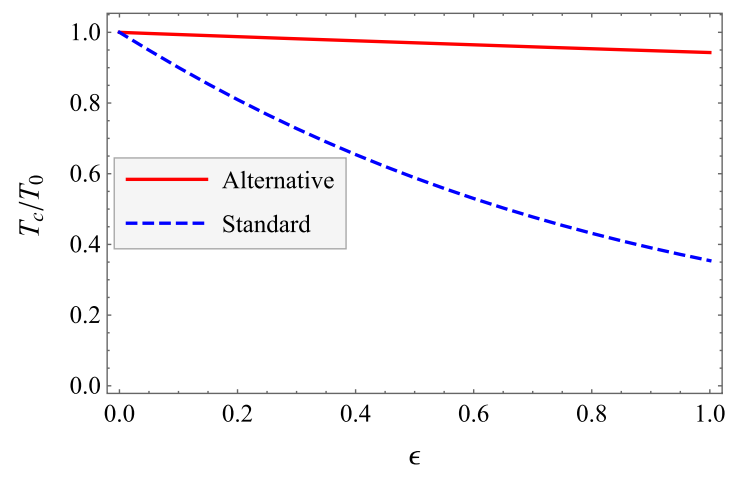

(b) Normalized critical temperature

FIG. 2. Absolute (a) and normalized (b) critical temperature for varying backreaction parameter $\epsilon$. $T_{0}$ is the critical temperature in the probe limit $\epsilon=0$. The critical temperature drops with increasing backreaction for both the standard and alternative quantization, indicating a suppression of the condensate mechanism.

domains lives on a rectangular grid, $(r, x) \in(0,1) \times$ $\left(-\frac{L_{x}}{2}, \frac{L_{x}}{2}\right)$. The resulting linear system is solved by lowerupper decomposition or other iterative techniques.

At a critical temperature $T=T_{c}$, an instability of the black brane to form charged scalar hair occurs. This instability corresponds to a phase transition in the boundary theory. We begin with a discussion of the critical temperature $T_{c}$ and its dependence on the backreaction as given by the backreaction parameter $\epsilon=2 \kappa_{4}^{2}$. To determine where our solutions become unstable, we have to determine the critical temperature for a given $\epsilon$. We do this by perturbing the Reissner-Nordström background (5) with the scalar field $\psi=\phi(r) e^{-i \omega_{p} t}$. At the onset of the instability, the frequency of the unstable mode crosses zero, $\omega_{p}=0$. The critical temperature $T_{c}$ itself is therefore found by looking for a static normalizable solution to the scalar equation of motion with $\omega_{p}=0$. The results are shown in Fig. 2. We find that in the alternative quantization $T_{c} / \mu$ is always higher than in the standard case, since the alternative boundary conditions allow for more modes to become unstable in the IR, i.e., in the deep interior of the space, in the sense that they violate the Breitenlohner-Freedman bound there [35]. Furthermore, in AdS/CFT the mass of the scalar field in the bulk determines the dimension of the operator in standard $\left(\Delta_{+}\right) /$alternative $\left(\Delta_{-}\right)$quantization via $\Delta_{ \pm}=\frac{d}{2} \pm \sqrt{\frac{d^{2}}{4}+m^{2} L^{2}}$. From the field-theoretic point of view, the dimension of the operator in the alternative quantization is always smaller than that in the standard case, $\Delta_{-}<\Delta_{+}{ }^{5}$. The operator with the smaller scaling dimension is more relevant in the RG sense, and its effects will be more pronounced already at larger values of the renormalization scale. Hence, this operator will condense at higher temperatures, explaining the larger $T_{c}$ in the alternative case.

${ }^{5}$ Equality is reached at the Breitenlohner-Freedman bound $m^{2} L^{2}=-d^{2} / 4$.
In relation to this, we find that for a value of the backreaction parameter in the range $\epsilon \in[0,1]$, the critical temperature for the alternative (BEC) case has only a milder dependence on $\epsilon$ compared to that in the standard (BCS) case. Within this range, the backreaction is relatively weak, and we can expand the blackening factor $f(z)$ in a power series in $\epsilon$ near the probe limit $\epsilon=0[6,19],{ }^{6}$ with the first correction being

$f(z)=1-\left(\frac{z}{z_{+}}\right)^{3}+\epsilon\left(\frac{3 \mu}{8 \pi T}\right)^{2}\left(\frac{z}{z_{+}}\right)^{3}\left(\frac{z}{z_{+}}-1\right)+\ldots$

Since $\epsilon$ is multiplied by $(\mu / T)^{2}$, just below the critical temperature the effect of backreaction is suppressed for higher $T_{c} / \mu$. Since $T_{c} / \mu$ is at least a factor of 4 larger in the alternative case, the backreaction-induced change in $f(z)$ in the alternative quantization is reduced by a factor of 16 compared to the standard case. This implies that in the alternative case, the equations of motions are far less sensitive to changes in the backreaction parameter. In particular, the change in $T_{c} / \mu$ to order $\epsilon$ is also suppressed compared to the standard case. In summary, the insensitivity of $T_{c} / \mu$ to the backreaction parameter in the alternative case is due to the enhancement of $T_{c} / \mu$ in the probe limit as compared to the standard case.

The previous argument is corroborated by the change in the scalar field profile $\Psi$ as shown for the scalar in Fig. 3, which is also found to be much weaker in the alternative quantization compared to the standard case. Also, the boundary condition of $\Psi$ on the horizon,

$\left.\frac{\partial_{\xi} \Psi}{\Psi}\right|_{\xi=1}=-\frac{8}{\epsilon(3 \mu / 4 \pi T)^{2}-12}+\ldots, \quad \xi=\frac{z}{z_{+}}$,

\footnotetext{
${ }^{6}$ A possible hyperscaling-violating factor has been ruled out by the construction of the IR fixed point in Ref. [35].
} 


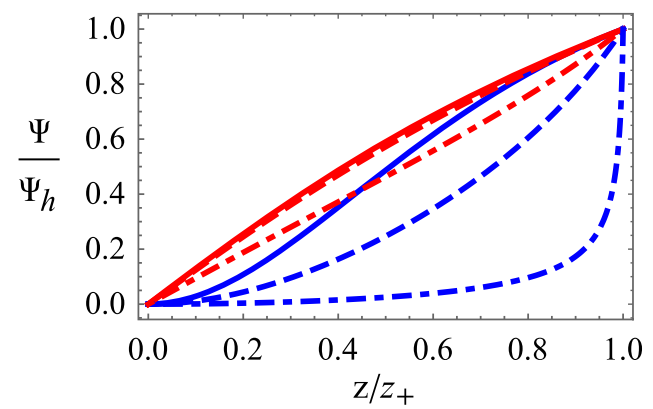

FIG. 3. Profiles of $\Psi / \Psi_{h}$ just below the critical temperature, where $\Psi_{h}$ is the value of $\Psi$ on the horizon. We show the cases of $\epsilon=0$ (solid line), $\epsilon=1$ (dashed line), and $\epsilon=10$ (dot-dashed line). The blue (red) line refers to the profile in the standard (alternative) quantization.

depends more mildly on $\epsilon$ in the alternative quantization. Thus, we see that in all cases $T_{c} / \mu$ in the probe limit controls the first correction in the backreaction parameter $\epsilon$, and hence in the alternative quantization it depends more weakly on the backreaction. However, in a wider range of $\epsilon$,

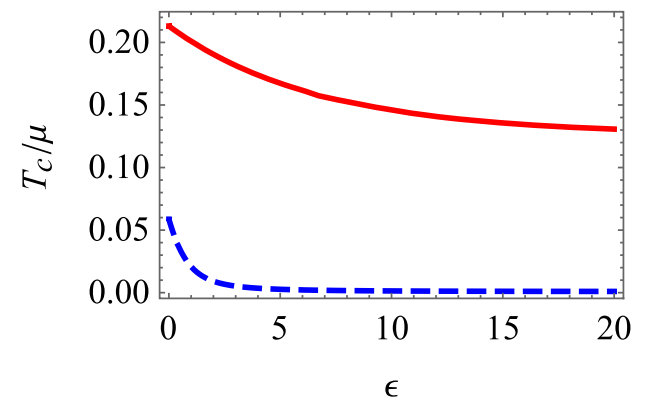

FIG. 4. $T_{c} / \mu$ in the standard quantization (blue) and alternative quantization (red).

the dependence on $\epsilon$ can only be found numerically, as shown in Fig. 4.

Based on the above results for the critical temperature, we now construct the solution with backreaction for $T<T_{c}$. These are hairy charged black holes dual to the superfluid phase. The seed configurations of the matter field can be chosen as $\psi_{ \pm} \sim \tanh \left(x-L_{x} / 4\right) \tanh \left(-x-L_{x} / 4\right)$. As a result, the components of the metric and the configuration of the matter fields are shown in Figs. 5

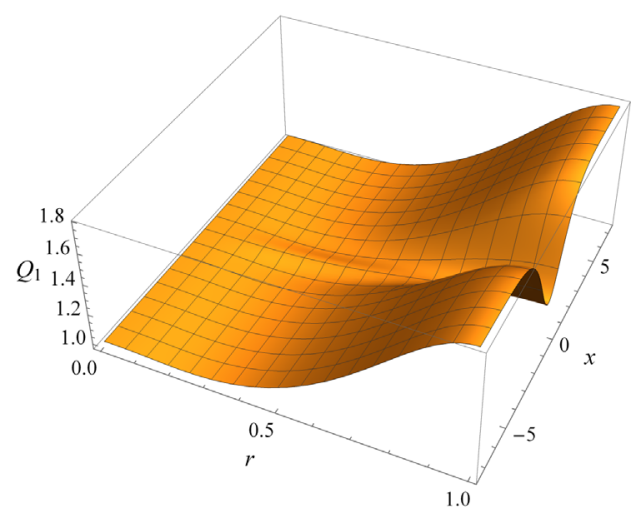

(a) $Q_{1}$

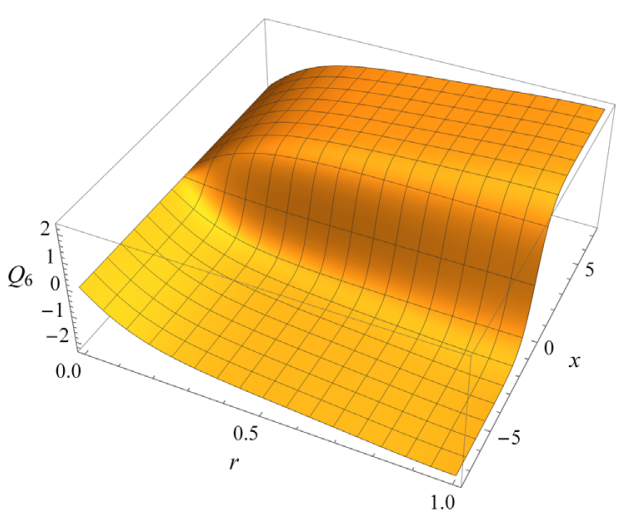

(c) $Q_{6}$

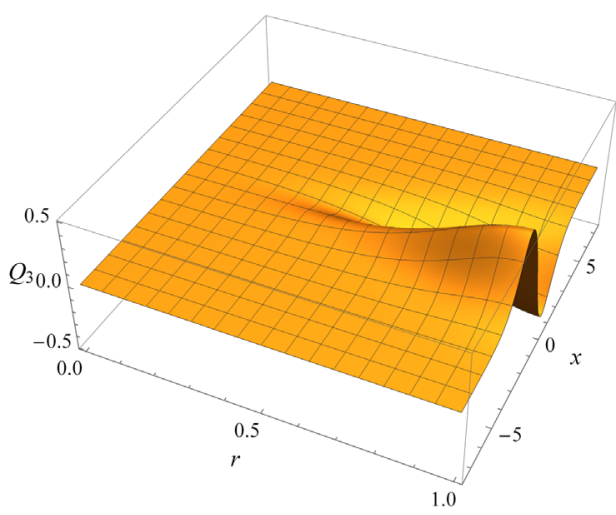

(b) $Q_{3}$

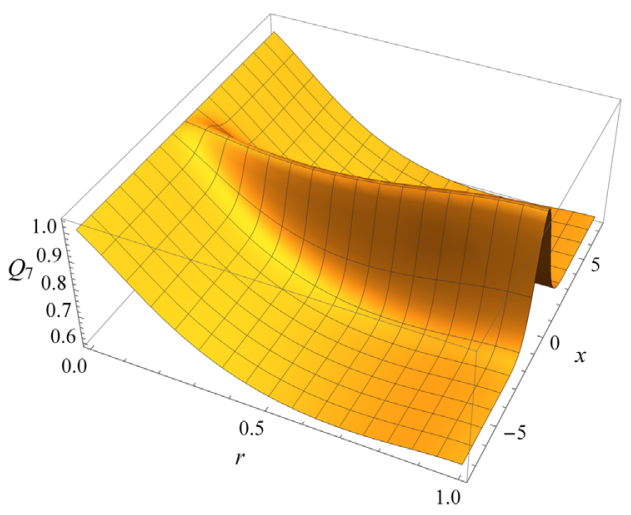

(d) $Q_{7}$

FIG. 5. The profile of (a) the function $Q_{1}$ setting the $t t$ metric component, (b) the function $Q_{3}$ setting, together with $Q_{4}$, the $r r$ metric component, (c) the function $Q_{6}$ setting the charged scalar profile, and (d) the function $Q_{7}$ setting the $t$ component of the gauge field, at $\epsilon=0.25, T / T_{c}=0.5$ in the standard quantization. 


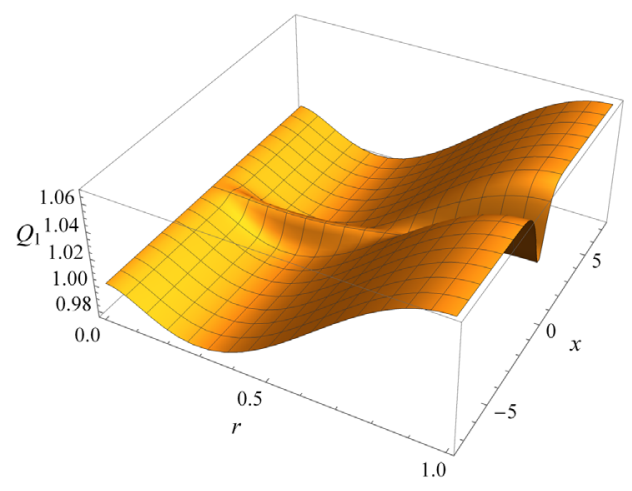

(a) $Q_{1}$

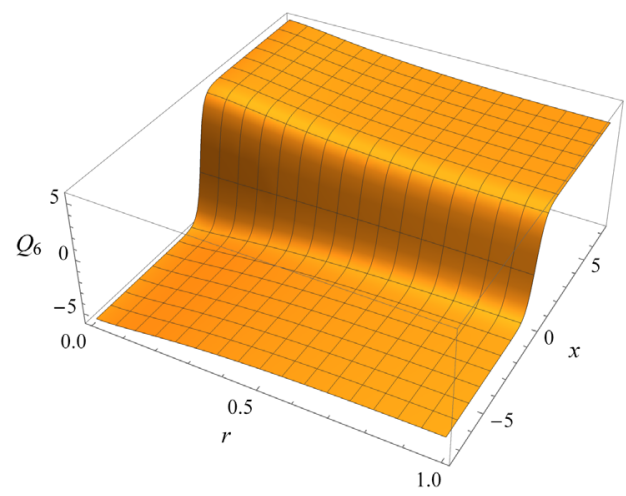

(c) $Q_{6}$

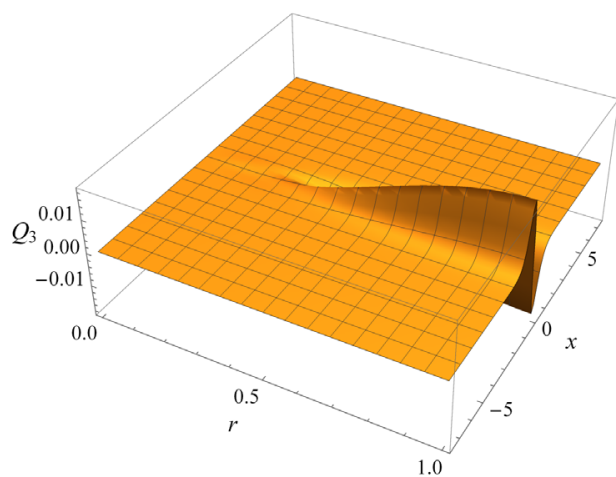

(b) $Q_{3}$

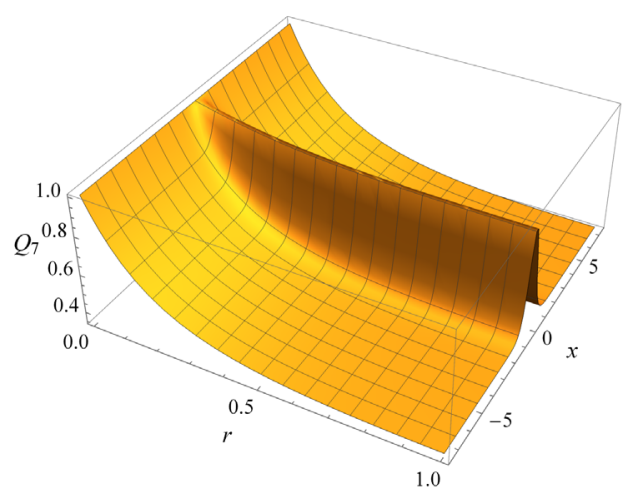

(d) $Q_{7}$

FIG. 6. The profile of (a) the function $Q_{1}$ setting the $t t$ metric component, (b) the function $Q_{3}$ setting, together with $Q_{4}$, the $r r$ metric component, (c) the function $Q_{6}$ setting the charged scalar profile, and (d) the function $Q_{7}$ setting the $t$ component of the gauge field, at $\epsilon=0.25, T / T_{c}=0.5$ in the alternative quantization.

and 6. We can see that larger fluctuations of the spacetime metric appear only near the core of the soliton.

From the asymptotic form of the matter fields, we then read off the expectation value of the charged condensate and the particle number density in the dual field theory. These are shown in Fig. 7. As found in Refs. [8,12], in the probe limit for the BEC superfluid the soliton shows a larger depletion fraction which is, however, smaller than $100 \%$. $^{7}$ We expect that the depletion will be close to $100 \%$ at lower temperature, where backreaction has to be included. The dependence of the depletion factor on temperature for different $\epsilon$ is plotted in Fig. 8. Contrary to expectations, for the BEC soliton the depletion in the core is considerably smaller than $100 \%$, and even lower than that in the probe limit at low temperature. As Fig. 9 shows, the depletion decreases as the backreaction increases. This behavior is observed in both the BCS and BEC regime of our holographic model. We give a

\footnotetext{
${ }^{7}$ One possible explanation for this finding is that the temperature here is not low enough. In fact, the solution with backreaction at lower temperature is still very unreliable and difficult to obtain using numerical methods.
}

qualitative interpretation of this behavior in terms of the balance between uncondensed and condensed charge in the boundary theory as the homogeneous and normal state is reached under the limit of large backreaction in the Appendix B. We think that the underlying reason for this behavior is the nature of the condensate zero-temperature IR fixed point, which may be uncharged. In the near future we plan to analyze this fixed point using analytic methods along the lines of Ref. [36], and also construct other fixed points which show increasing depletion with decreasing temperature.

\section{A SIMPLE MECHANISM FOR THE SNAKE INSTABILITY}

In this section we turn to the discussion of the thermodynamics of our holographic dark soliton solution, as well as its instabilities. Since the boundary chemical potential and temperature are fixed, our system is in the grand canonical ensemble characterized by the grand potential

$$
\Omega=E-T S-\mu N .
$$




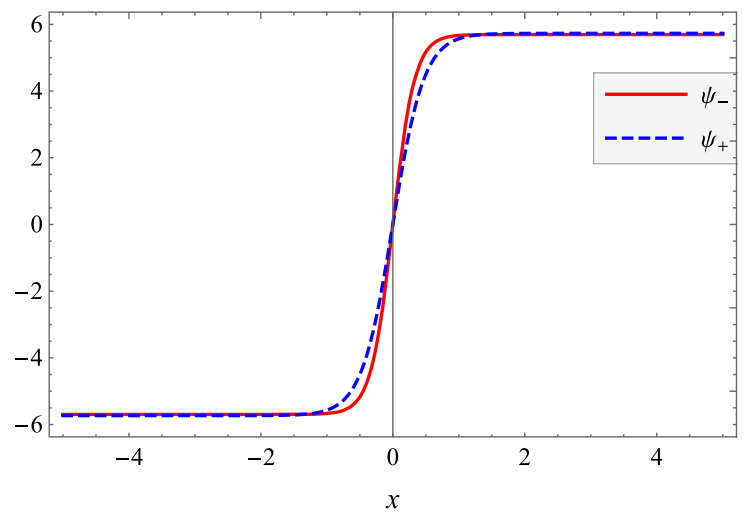

(a) Condensate (order parameter)

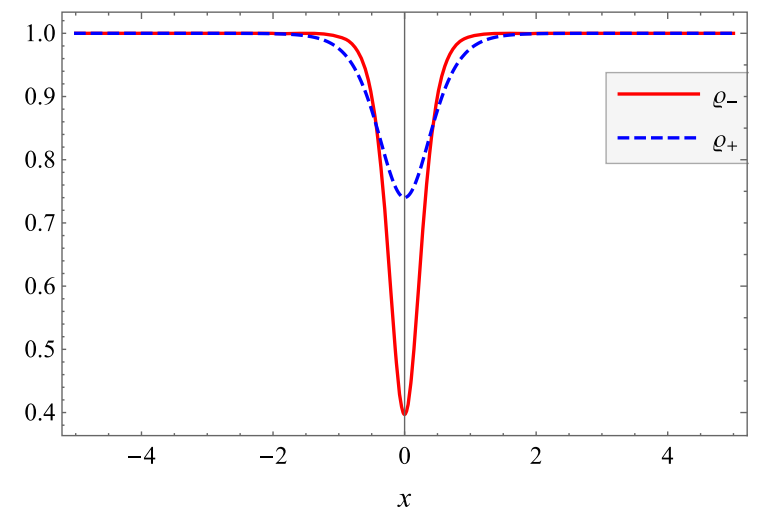

(b) Particle number density

FIG. 7. The condensate (a) and the particle number density (b) as a function of $x$ at $\epsilon=0.25, T / T_{c}=0.5$. $Q_{+}$and $Q_{-}$are the particle number densities normalized with their equilibrium values at $x \rightarrow \pm \infty$. Red solid lines correspond to the alternative (BEC) case, while blue dashed lines correspond to the standard (BCS) case. The charge depletion is considerably larger in the BEC case compared to the BCS case.

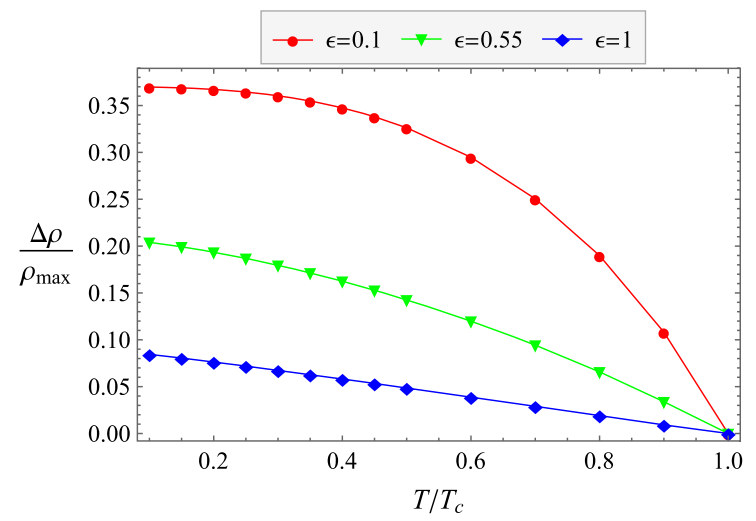

(a) Standard (BCS) case

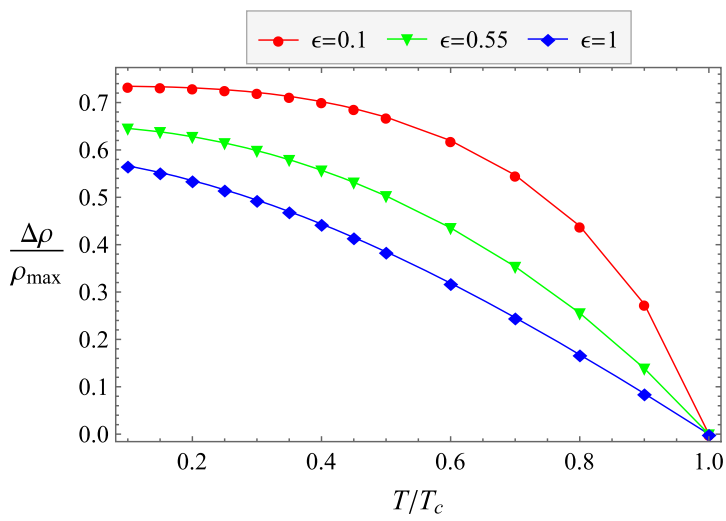

(b) Alternative (BEC) case

FIG. 8. The depletion of the particle number density as a function of $T / T_{c}$ for different $\epsilon$ in the (a) standard (BCS) and (b) alternative (BEC) case. In both cases, the depletion factor decreases with increasing backreaction, i.e., more charge is present at the soliton core.

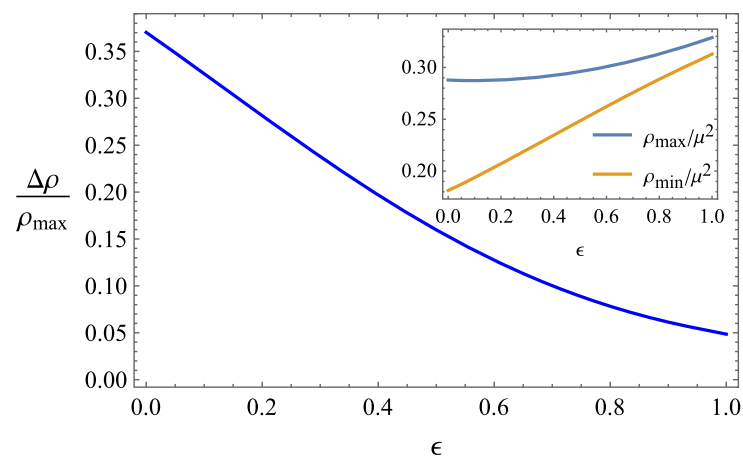

(a) Standard (BCS) case

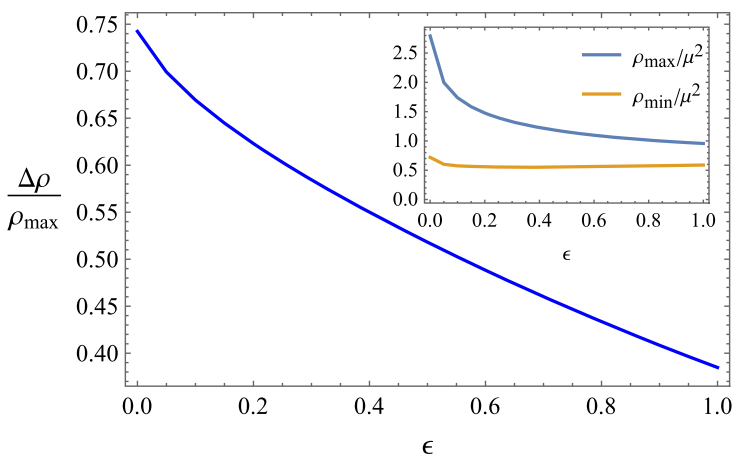

(b) Alternative (BEC) case

FIG. 9. The depletion of the particle number density as a function of $\epsilon$ at $T / T_{c}=0.5$. The inset panel shows the change in the particle number density far away from the core of the soliton and at the core. In both cases, increasing backreaction reduces the charge depletion. 


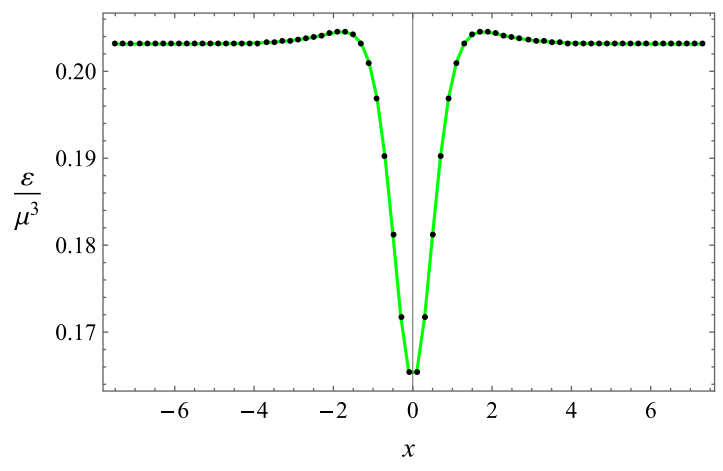

(a) Total energy density

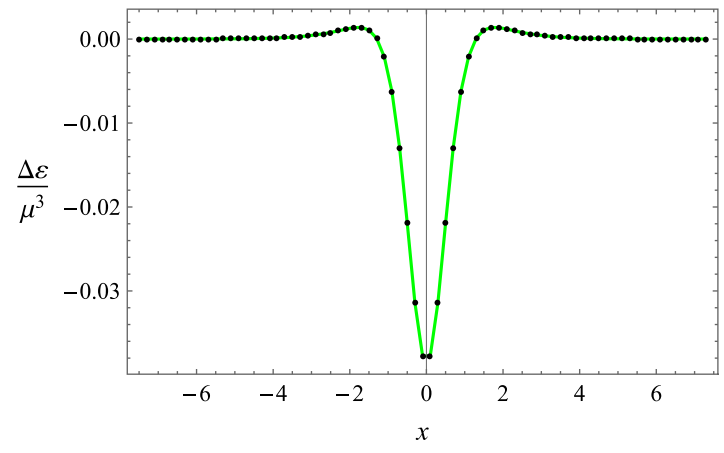

(b) Subtracted energy density

FIG. 10. Total (a) and subtracted (b) energy density at $\epsilon=0.25, T=0.5 T_{c}$. The subtracted energy density, which can be interpreted as the effective mass density of the soliton in the condensate phase, is found to be negative.

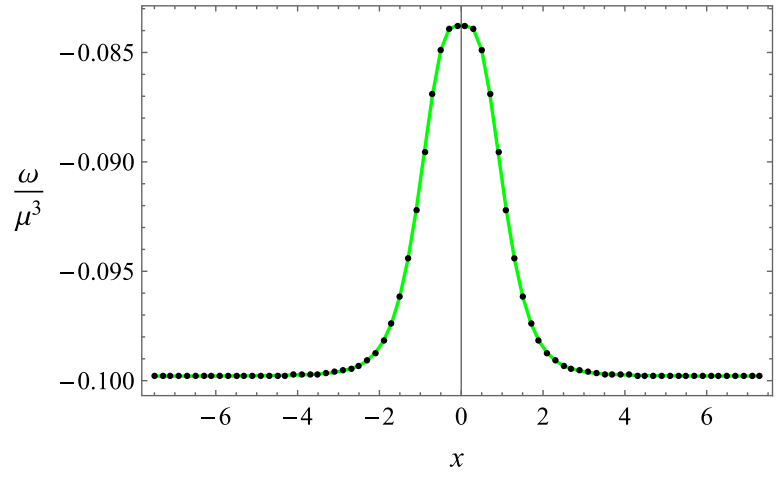

(a) Total grand potential density

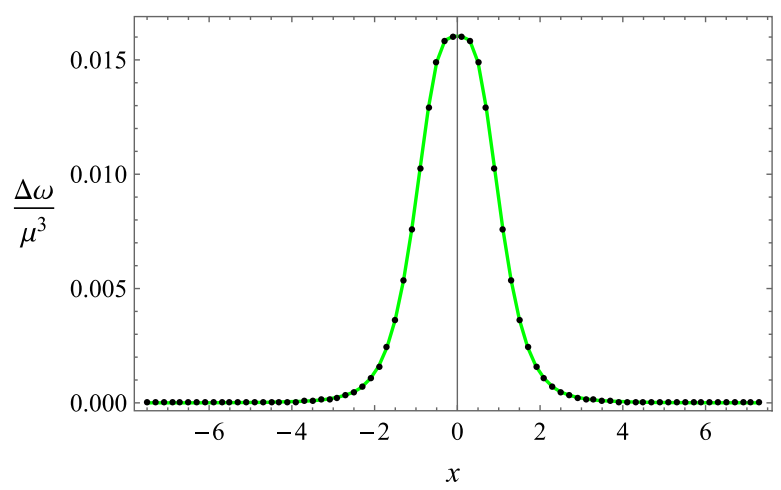

(b) Subtracted grand potential density

FIG. 11. Total (a) and subtracted (b) grand potential density at $\epsilon=0.25, T=0.5 T_{c}$. After integration over the width of the soliton, the latter becomes the surface tension of the soliton, which is found to be positive.

Here $N$ is the total particle number obtained by integrating the charge density $\rho$ over space. The internal energy is found to be

$$
E=\int_{\Sigma_{t}} d^{2} x \sqrt{\eta}\left[T_{\mu \nu}\left(\partial_{t}\right)^{\mu}\right] t^{\nu}
$$

Here $\eta_{\mu \nu}$ is the induced metric on the surface $\Sigma_{t}$ at $z=0$ and $t=$ const, with unit normal vector $t^{\nu}$, and $T_{\mu \nu}$ is the holographic stress-energy tensor; see Appendix A for a detailed calculation. $S$ is the usual black hole entropy, given by

$$
S=\frac{A_{h}}{4 G}=\frac{4 \pi z_{h}^{2}}{\epsilon} \int \sqrt{Q_{4}(0, x) Q_{5}(0, x)} d^{2} x .
$$

Since the soliton extends in a noncompact spatial direction, in what follows we will consider densities of the above thermodynamic quantities. In particular, the grand potential $\Omega$ and the energy $E$ are replaced by their respective densities $\omega$ and $\varepsilon$. Far away from the soliton center, these local quantities will approach their homogeneous equilibrium values. Therefore, the soliton core is characterized by the difference between these local densities and their equilibrium values.

The energy density difference is displayed in Fig. 10(b), where we see an obvious energy depletion around the soliton core. ${ }^{8}$ Upon integration along $x$, this depletion yields a negative effective energy difference $\Delta E=-7.220$ (in units of the chemical potential $\mu$ ). This energy difference $\Delta E$ can be seen to set the effective mass of the soliton, which is negative, as expected for a dark soliton [22].

The grand potential density difference is plotted in Fig. 11(b), from which we see that there is a grand potential cost for the soliton with respect to the homogeneous background. Upon integration along $x$, the grand potential cost of the soliton yields the surface tension coefficient of the soliton. The surface tension coefficient $\sigma$ of a domain wall such as the soliton is defined as the external work $W$ necessary to enlarge the surface by a unit area while keeping temperature and chemical potential fixed. Under

\footnotetext{
${ }^{8}$ In this section, we only show the explicit results for the soliton in the BCS superfluid, while the results for the BEC case are similar.
} 


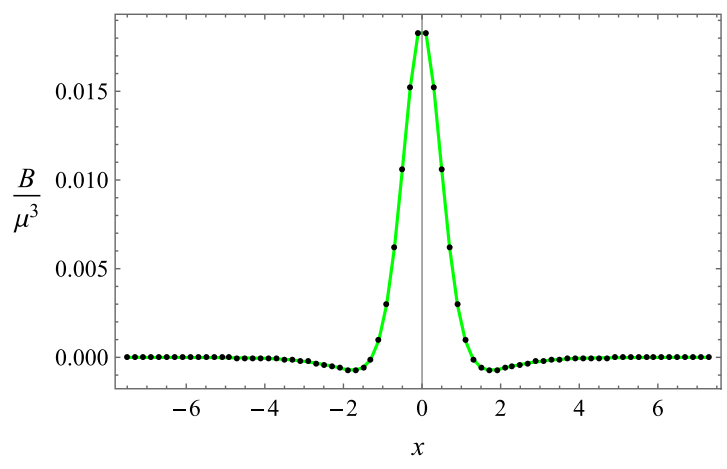

(a) Pressure anisotropy

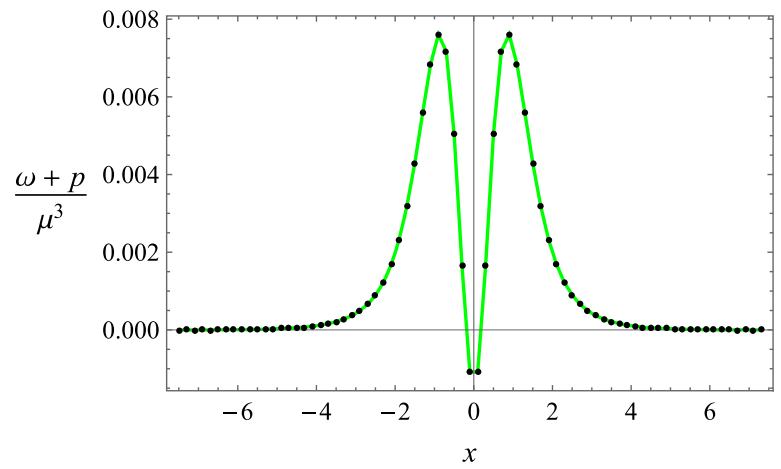

(b) Validity of the thermodynamic relation

$$
\omega=-p
$$

FIG. 12. Pressure anisotropy (a) and thermodynamical relation (b) at $\epsilon=0.25, T=0.5 T_{c}$. Panel (b) in particular shows that the system is in a thermalized state away from the soliton core.

these conditions, the external work is just the increase of the grand potential due to the enlargement of the domain wall surface, $W=\Omega-\Omega_{0}$, with $\Omega_{0}$ being the grand potential of the corresponding homogeneous system without the domain wall. For the case displayed in Fig. 11(b), we numerically determine the surface tension in units of the chemical potential to be $\sigma=6.615$. As a consistency check for our numerics, we also plot the pressure anisotropy $B \equiv p_{x}-p$ in Fig. 12(a) and check the thermodynamic relation $\omega=-p$, where $p$ is the average pressure, which should hold far away from the soliton center.

With the results for these thermodynamic variables, we can confirm the following explanation for the so-called snake instability of the dark soliton [23]. The soliton moves through the condensate as a heavy, i.e., nonrelativistic particle [22]. Since it has a negative effective mass $M_{\text {eff }}=\Delta E<0$, its energy $E_{s}=\frac{M_{e} f f}{2} \dot{q}^{2}$ decreases with increasing velocity $\dot{q}$. As shown in Ref. [22], for a homogeneous solitonic configuration, the velocity grows exponentially. This is the so-called self-acceleration instability [22] of the dark soliton. The self-acceleration originates from the dissipative interaction of the soliton with the surrounding condensate. As discussed in Ref. [22], for a homogeneous soliton configuration the selfacceleration terminates once the soliton velocity reaches the speed of sound, at which point the soliton decays into sound waves that dissipate away in the condensate.

On the other hand, the soliton has a positive surface tension coefficient $\sigma$. As shown in the hydrodynamic approximation in Ref. [23], the combined effect of the negative effective mass together with the positive surface tension leads to a growing transverse bending mode with a finite wave vector. The same instability was also found in the holographic quasinormal mode spectrum in Ref. [31]. Once this transverse bending mode starts to grow, the selfacceleration instability enhances the local bending, leading to the formation of a snake-like structure.

\section{CONCLUSIONS AND DISCUSSIONS}

We investigated the implications of including the gravitational backreaction onto solitons in holographic superfluid systems. We numerically solved the Einstein equations coupled with the relevant matter fields. As compared to the probe limit, and contrary to our original expectations, increasing the backreaction decreases the depletion of the particle number density in the soliton core. We gave a qualitative interpretation of this in terms of the balance of the ratios of condensate and noncondensate over total charge in the dual field theory as a homogeneous state is reached at strong backreaction. Finally, we computed the holographic stress-energy tensor of the system and confirmed a simple holographic explanation for the snake instability of the dark soliton.

In this work, we restricted ourselves to the asymptotic regimes of BEC and BCS superfluidity. In particular, we did not investigate the Robin boundary conditions necessary to model the actual unitary regime, in which the strongly coupled unitary fermion system is expected to live. We plan to investigate the behavior of the soliton in the crossover regime in future work. Such an investigation may in particular provide a better description of the intermediate unitary fermion regime, for which the Bogoliubov-de Gennes theory provides only a broad approximation [23]. Furthermore, our qualitative interpretation of the reduction of the depletion factor implicitly relies on the assumption of a noncondensate homogeneous infrared fixed point at strong backreaction or low temperatures. In our holographic superfluid strong backreaction implies a smaller critical temperature, and hence the limit of strong backreaction is equivalent to the limit of low temperatures. Only if the homogeneous infrared fixed point is noncondensate, i.e., the condensing operator is irrelevant at the infrared fixed point, can the system return to the noncondensate state in the low-temperature limit. We will investigate the possible infrared fixed points in our system 
along the lines of Ref. [36] to support our qualitative interpretation in future work. In the thin-domain-wall limit of the soliton, an analytic treatment of the domain wall in terms of a brane with junction conditions is also conceivable.

\section{ACKNOWLEDGMENTS}

We would like to thank Xin Li, Hong Liu, Jie Ren, Ya-Wen Sun, Xiaoning Wu, and Hongbao Zhang for useful discussions. In particular, Zhongshan Xu is grateful to Jie Ren for his explanations concerning the DeTurck method. This work is partially supported by the NSFC with Grants No. 11975235 and 11847229. Y. D. is supported by the China Scholarship Council. Y. D., J. E., and R. M. acknowledge financial support through the Deutsche Forschungsgemeinschaft (DFG, German Research Foundation), project-id 258499086SFB 1170 "ToCoTronics," and through the WürzburgDresden Cluster of Excellence on Complexity and Topology in Quantum Matter-ct.qmat (EXC 2147, project-id 39085490). Y. T. is also supported by the "Strategic Priority Research Program of the Chinese Academy of Sciences" with Grant No. XDB23030000. Z.-Y.X. also acknowledges the support from the National Postdoctoral Program for Innovative Talents BX20180318, funded by the China Postdoctoral Science Foundation.

\section{APPENDIX A: HOLOGRAPHIC STRESS-ENERGY TENSOR}

In order to compute the holographic stress-energy tensor, following the process of Refs. [37,38], we need to find the asymptotic expansion of the metric at the conformal boundary. The expansion is obtained by solving Eq. (17) with boundary conditions order by order in $(1-r)$ and in addition imposing $\xi^{\mu}=0$,

$$
\begin{gathered}
Q_{i}(r, x)=1-\frac{\epsilon Q_{6}^{(0)}(x)}{z_{h}^{2}}(1-r)^{2}+q_{i}(x)(1-r)^{3} \\
+O\left[(1-r)^{4}\right], \quad i=1,4,5, \quad(\mathrm{~A} 1 \mathrm{a}) \\
Q_{2}(r, x)=1+\frac{8 \epsilon Q_{6}^{(0)}(x) Q_{6}^{(1)}(x)}{3 z_{h}^{2}}(1-r)^{3}+O\left[(1-r)^{4}\right], \\
Q_{3}(r, x)=\frac{2 \epsilon Q_{6}^{(0)}(x) \partial_{x} Q_{6}^{(0)}(x)}{z_{h}^{3}}(1-r)^{3}+O\left[(1-r)^{4}\right], \\
Q_{6}(r, x)=Q_{6}^{(0)}(x)+Q_{6}^{(1)}(x)(1-r)+\cdots, \quad(\mathrm{A} 1 \mathrm{~b}) \\
Q_{7}(r, x)=Q_{7}^{(0)}(x)+Q_{7}^{(1)}(x)(1-r)+\cdots .
\end{gathered}
$$

Here $q_{1}(x), q_{4}(x)$, and $q_{5}(x)$ satisfy Eqs. (A3a)-(A3b) related to the tracelessness and conservation of the boundary stress-energy tensor,

$$
T_{i}^{i}=0, \quad \partial_{i} T^{i j}=0 .
$$

Using these relations, one can explicitly show the following conditions:

$$
\begin{aligned}
& q_{1}(x)+q_{4}(x)+q_{5}(x) \\
& =-\frac{\epsilon Q_{6}^{(0)}(x)\left(-3 Q_{6}^{(0)}(x)+8 Q_{6}^{(1)}(x)\right)}{z_{h}^{2}},
\end{aligned}
$$

$$
\partial_{x} q_{4}(r, x)=-\frac{2 \epsilon\left[\left(-3 Q_{6}^{(0)}(x)+8 Q_{6}^{(1)}(x)\right) \partial_{x} Q_{6}^{(0)}(x)+2 Q_{6}^{(0)}(x) \partial_{x} Q_{6}^{(1)}(x)\right]}{3 z_{h}^{2}}
$$

Having obtained the asymptotic behavior of the metric functions, one then changes to Fefferman-Graham coordinates $(z, v)$ by an expansion of the series (A4a) and demands $g_{z z}=\frac{1}{z^{2}}$ and $g_{z v}=0$ to determine the two functions $\left\{a_{k}(v), b_{k}(v)\right\}$ order by order in $z$. Here we provide the first few terms necessary for the computation of the holographic stress-energy tensor:

$$
\begin{gathered}
\left\{\begin{array}{l}
r=1-\frac{z_{h}}{2} z+\sum_{k=2}^{\infty} a_{k}(v) z^{k}, \\
x=v+\sum_{k=1}^{\infty} b_{k}(v) z^{k},
\end{array}\right. \\
a_{2}(v)=-\frac{z_{h}^{2}}{8}, \quad a_{3}(v)=-\frac{z_{h}^{3}}{16},
\end{gathered}
$$

$$
\begin{gathered}
a_{4}(v)=\frac{z_{h}^{2}\left(24 \epsilon \mu^{2}+51 z_{h}^{2}+32 \epsilon Q_{6}^{(0)}(v) Q_{6}^{(1)}(v)\right)}{1152}, \\
b_{1}(v)=b_{2}(v)=b_{3}(v)=0, \\
b_{4}(v)=-\frac{\epsilon Q_{6}^{(0)}(v) \partial_{v} Q_{6}^{(0)}(v)}{16} .
\end{gathered}
$$

Finally, the holographic stress-energy tensor is computed by using Eq. (A5a) in the standard quantization and Eq. (A5b) in the alternative quantization $[39,40]$,

$$
T_{\mu \nu}=\frac{1}{\kappa_{4}^{2}} \lim _{z \rightarrow 0} \frac{1}{z}\left(K_{\mu \nu}-\gamma_{\mu \nu} K-2 \gamma_{\mu \nu}-\frac{\epsilon}{2}|\Psi|^{2} \gamma_{\mu \nu}\right),
$$




$$
\begin{aligned}
T_{\mu \nu}= & \frac{1}{\kappa_{4}^{2}} \lim _{z \rightarrow 0} \frac{1}{z}\left[K_{\mu \nu}-\gamma_{\mu \nu} K-2 \gamma_{\mu \nu}\right. \\
& \left.+\frac{\epsilon}{2}\left(-\Psi^{\dagger} n^{\sigma} D_{\sigma} \Psi-\text { C.C. }+|\Psi|^{2}\right) \gamma_{\mu \nu}\right] .
\end{aligned}
$$

Here $K_{\mu \nu}$ is the extrinsic curvature associated with an inward-pointing unit normal vector $n^{\sigma}$ on the constant $z=\epsilon$ surface near the boundary. $\gamma_{\mu \nu}$ is the induced metric on the cutoff surface. The last term in Eq. (A5) cancels the divergences due to the presence of the scalar field [41].

\section{APPENDIX B: PARTICLE NUMBER (CHARGE) CHANGE WITH BACKREACTION}

Here we give a qualitative interpretation of the depletion that decreases with increasing backreaction, in terms of the balance between uncondensed and condensed charge in the

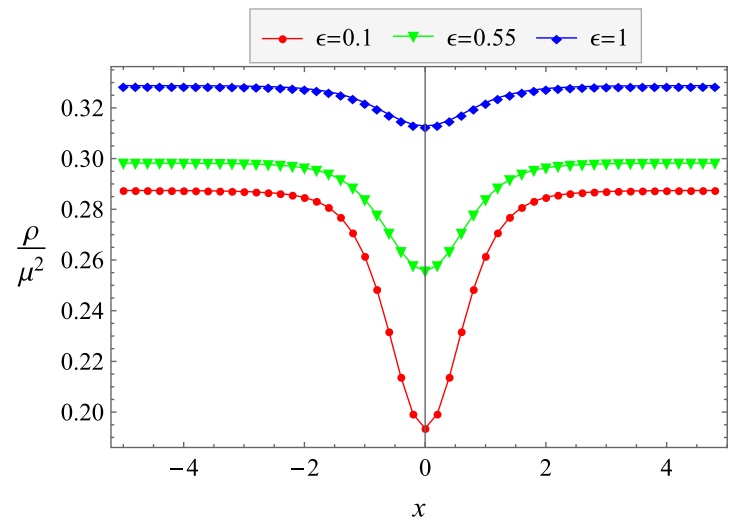

(a) Total particle number density.

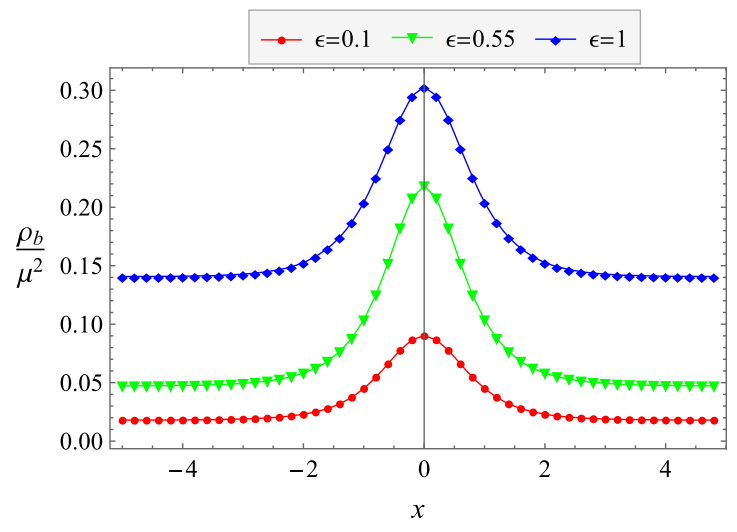

(c) Uncondensed particle number boundary theory as the homogeneous and normal state is reached under the limit of large backreaction. For our static but inhomogeneous charged scalar and gauge field configuration, by integrating the $t$ component of the Maxwell equations (4) over the holographic $r$ coordinate one obtains the total particle number density in terms of three contributions,

$$
\begin{aligned}
\left.\sqrt{-g} F^{\mathrm{tr}}\right|_{r=1}= & \left.\sqrt{-g} F^{\mathrm{tr}}\right|_{r=0}+\int_{0}^{1}\left(-\sqrt{-g} J^{t}\right) d r \\
& +\int_{0}^{1} \partial_{x} \sqrt{-g} F^{x t} d r, \\
J^{\nu}= & i g^{\mu \nu}\left[\Psi^{\dagger}\left(D_{\mu} \Psi\right)-\Psi\left(D_{\mu} \Psi\right)^{\dagger}\right] .
\end{aligned}
$$

On the right-hand side of Eq. (B1) the first term yields the uncondensed particle number density, given by the electric flux evaluated at the horizon. The second term is the

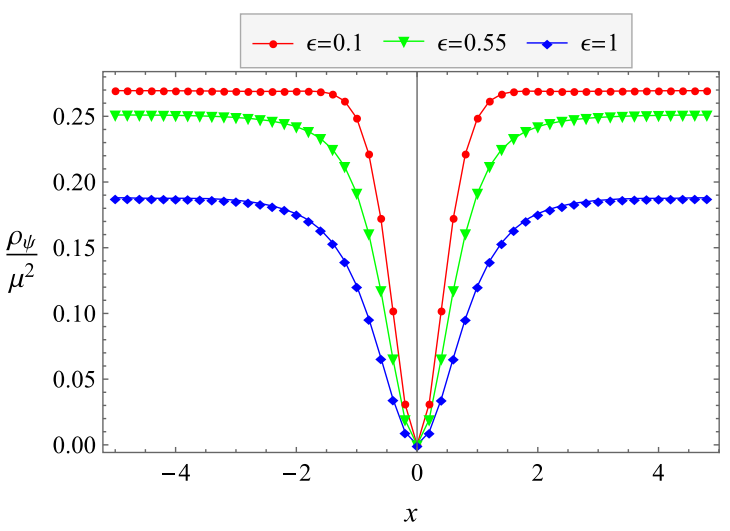

(b) Condensed particle number density.

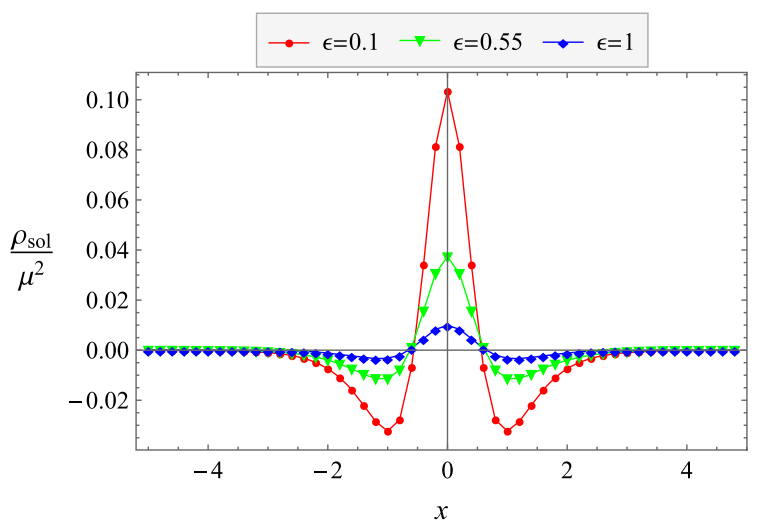

(d) Soliton contribution.

density.

FIG. 13. Change of the total charge density (a), condensed charge density (b), uncondensed charge density (c), and soliton contribution (d) with backreaction at $T=0.5 T_{c}$ in the standard (BCS) case. 


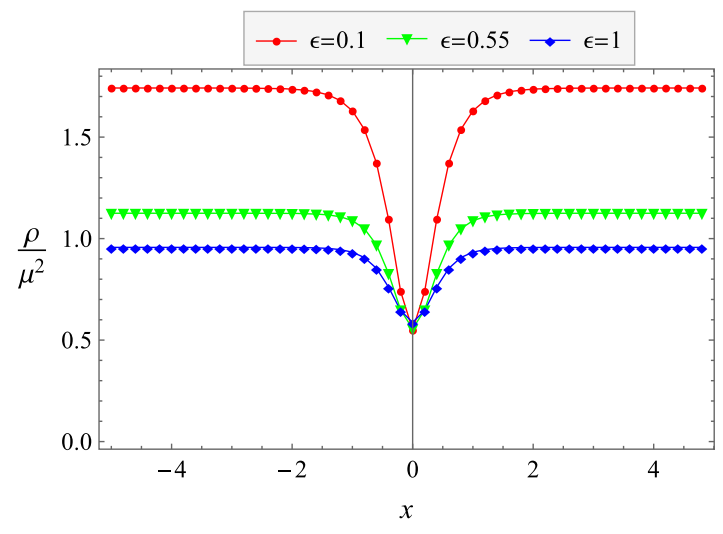

(a) Total particle number density.

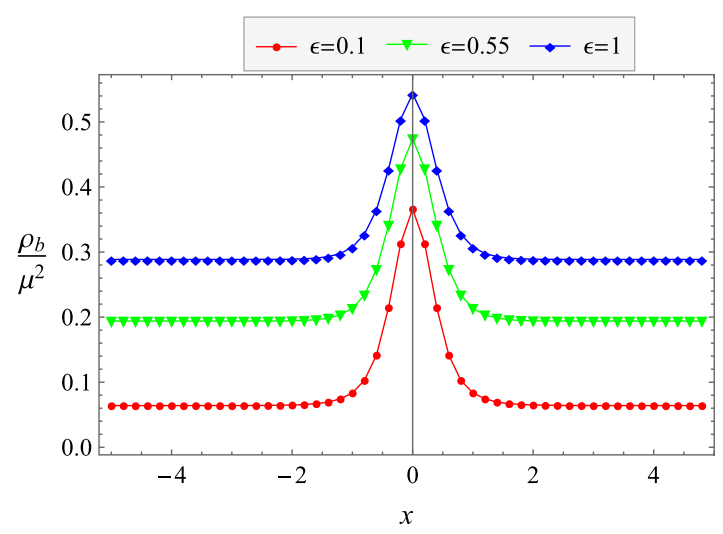

(c) Uncondensed particle number density.

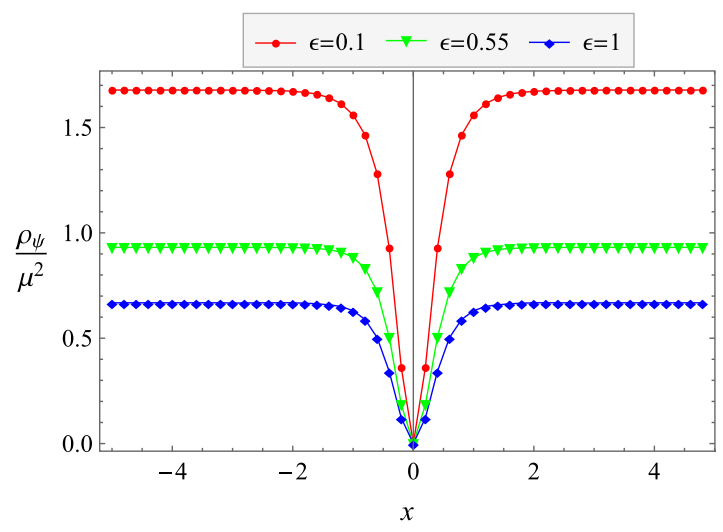

(b) Condensed particle number

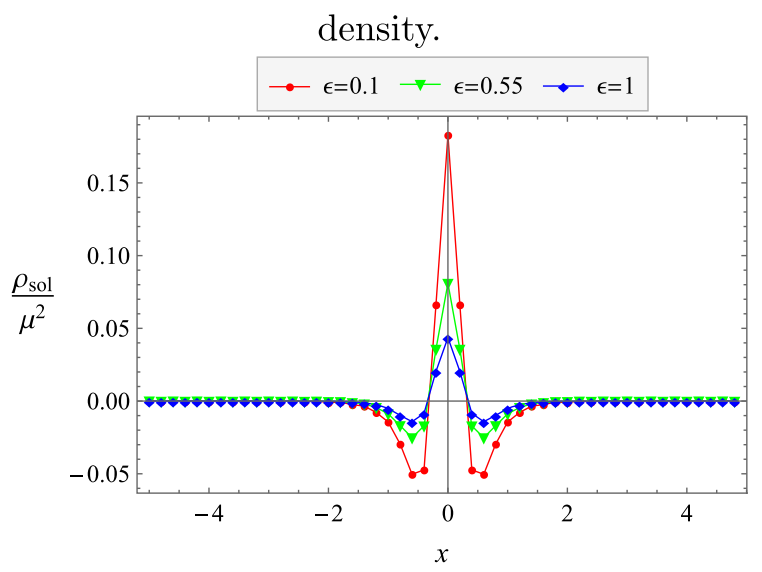

(d) Soliton contribution.

FIG. 14. Change of the total charge density (a), condensed charge density (b), uncondensed charge density (c), and soliton contribution (d) with backreaction at $T=0.5 T_{c}$ in the alternative (BEC) case.

condensed particle number density. The third term is a contribution arising from the inhomogeneous configuration of the soliton,

$$
\begin{aligned}
\underbrace{\left.\int d^{2} x \sqrt{-g} F^{\mathrm{tr}}\right|_{r=1}}_{N}= & \underbrace{\left.\int d^{2} x \sqrt{-g} F^{\mathrm{tr}}\right|_{r=0}}_{N_{b}} \\
& +\underbrace{\int d^{2} x \int_{0}^{1}\left(-\sqrt{-g} J^{t}\right) d r}_{N_{\psi}}
\end{aligned}
$$

A relation between the total charge $N$, uncondensed charge $N_{b}$, and condensed charge $N_{\psi}$ can be obtained by integrating Eq. (B1) over the spatial part. ${ }^{9}$ After integration,

\footnotetext{
${ }^{9}$ The spatial domain of all integrations above is $\left(-\frac{L_{x}}{2}, \frac{L_{x}}{2}\right) \times$ $\left(-\frac{L_{y}}{2}, \frac{L_{y}}{2}\right)$. Due to the translation symmetry along the $y$ direction, we normalize these charges with regard to $L_{y}$.
}

the soliton contribution in Eq. (B1) becomes a total derivative and hence vanishes if we integrate over a symmetric interval around $x=0$. The remaining terms in Eq. (B1) yield the relation (B3), in which we denote the three terms in order as $N, N_{b}$, and $N_{\psi}$. The ratios of the uncondensed and condensed charge over the total charge then have to add up to one,

$$
\frac{N_{b}}{N}+\frac{N_{\psi}}{N}=1
$$

As can be seen from Figs. 13 and 14, the backreaction suppresses the condensed particle density and promotes the uncondensed particle number density. On the other hand, from Fig. 15 the condensate (order parameter) far away from the soliton core also decreases strongly with increasing backreaction. It seems that the system returns to a homogeneous noncondensate state in the limit of large backreaction. This is, in particular, obvious for the BEC case [Fig. 15(b)], but the trend is also obvious for the BCS 


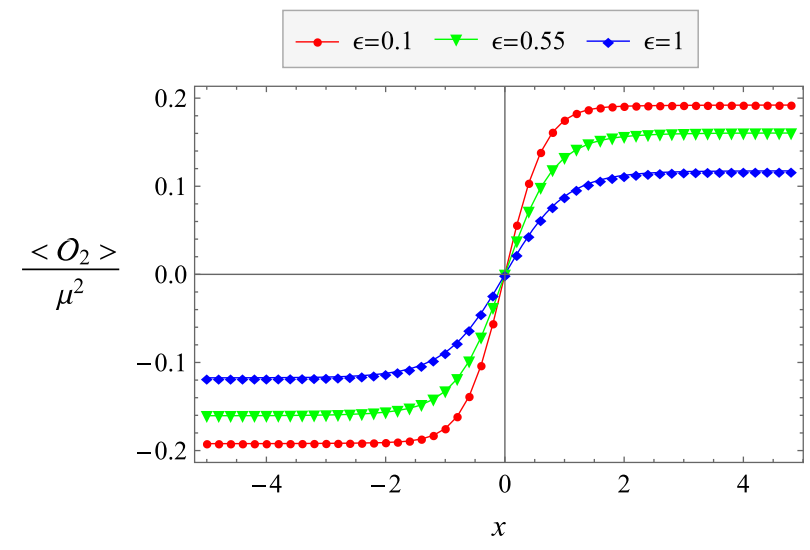

(a) Standard (BCS) case

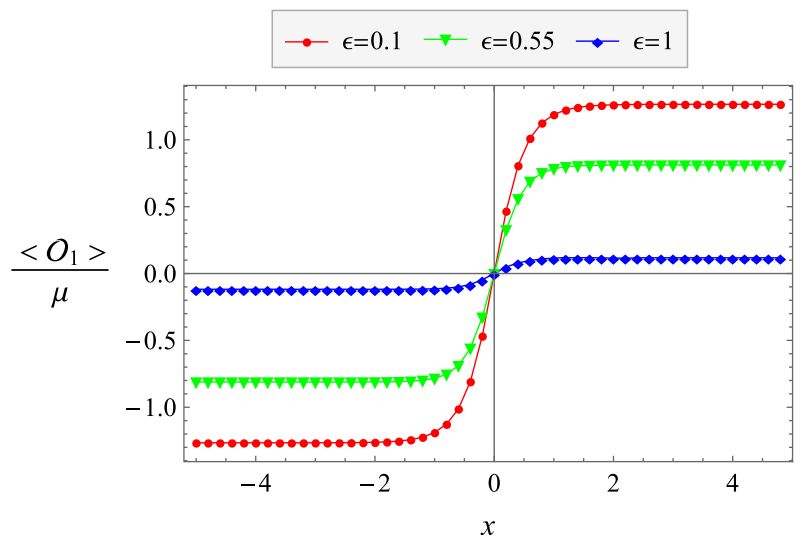

(b) Alternative (BEC) case

FIG. 15. The condensate as a function of $\epsilon$ at $T / T_{c}=0.5$.

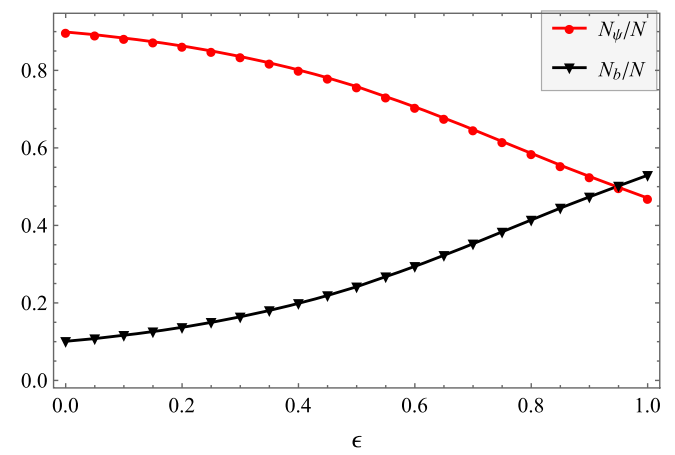

(a) Standard (BCS) case

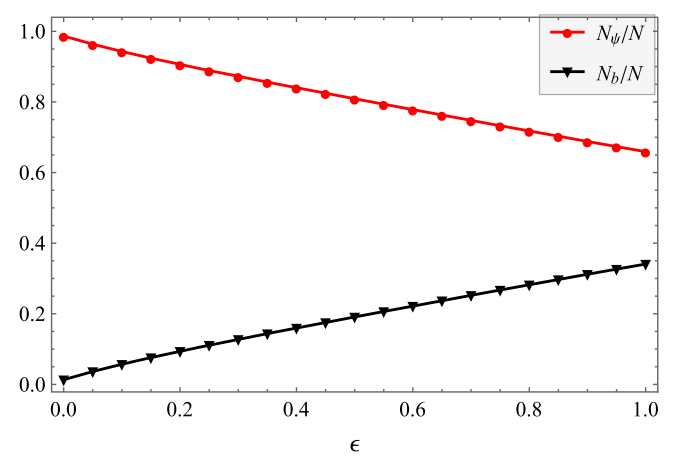

(b) Alternative (BEC) case

FIG. 16. The ratio of the two kinds of particle number as a function of $\epsilon$ at $T / T_{c}=0.5$.

case [Fig. 15(a)]. Furthermore, this interpretation of the data is consistent with the general expectation that backreaction inhibits the formation of the condensate in a holographic superconductor [42]. The suppression of the condensate implies that the ratio of condensate to total charge monotonically decreases with increasing backreaction. This can be seen from Fig. 16. Since the ratios of the condensate and noncondensate charge over the total charge are bound to add up to one by charge conservation, the ratio of noncondensate to total charge hence must increase with increasing backreaction. Taking these two facts together, we deduce that the depletion at the soliton core must decrease and vanish as the system approaches a homogeneous and normal state.
[1] J. Maldacena, The large N limit of superconformal field theories and supergravity, Adv. Theor. Math. Phys. 2, 231 (1998).

[2] E. Witten, Anti de sitter space and holography, Adv. Theor. Math. Phys. 2, 253 (1998).

[3] S. Gubser, I. Klebanov, and A. Polyakov, Gauge theory correlators from non-critical string theory, Phys. Lett. B 428, 105 (1998).

[4] M. Ammon and J. Erdmenger, Gauge/Gravity Duality (Cambridge University Press, Cambridge, England, 2015).
[5] C. P. Herzog, P. K. Kovtun, and D. T. Son, Holographic model of superfluidity, Phys. Rev. D 79, 066002 (2009).

[6] S. A. Hartnoll, C. P. Herzog, and G. T. Horowitz, Building a Holographic Superconductor, Phys. Rev. Lett. 101, 031601 (2008).

[7] M. Randeria and E. Taylor, Crossover from bardeencooper-schrieffer to bose-einstein condensation and the unitary fermi gas, Annu. Rev. Condens. Matter Phys. 5, 209 (2014). 
[8] V. Kernen, E. Keski-Vakkuri, S. Nowling, and K. P. Yogendran, Inhomogeneous structures in holographic superfluids. i. dark solitons, Phys. Rev. D 81, 126011 (2010).

[9] V. Kernen, E. Keski-Vakkuri, S. Nowling, and K. P. Yogendran, Inhomogeneous structures in holographic superfluids. ii. vortices, Phys. Rev. D 81, 126012 (2010).

[10] D. J. Frantzeskakis, Dark solitons in atomic bose-einstein condensates: from theory to experiments, J. Phys. A 43, 213001 (2010).

[11] M. Antezza, F. Dalfovo, L. P. Pitaevskii, and S. Stringari, Dark solitons in a superfluid fermi gas, Phys. Rev. A 76, 043610 (2007).

[12] V. Kernen, E. Keski-Vakkuri, S. Nowling, and K. P. Yogendran, Solitons as probes of the structure of holographic superfluids, New J. Phys. 13, 065003 (2011).

[13] V. Kernen, E. Keski-Vakkuri, S. Nowling, and K. P. Yogendran, Dark solitons in holographic superfluids, Phys. Rev. D 80, 121901(R) (2009).

[14] O. DeWolfe, O. Henriksson, and C. Wu, A holographic model for pseudogap in BCS-BEC crossover (i): Pairing fluctuations, double-trace deformation and dynamics of bulk bosonic fluid, Ann. Phys. (Amsterdam) 387, 75 (2017).

[15] E. Witten, Multi-trace operators, boundary conditions, and ads/cft correspondence, arXiv:hep-th/0112258v3.

[16] T. Hartman and L. Rastelli, Double-trace deformations, mixed boundary conditions and functional determinants in AdS/CFT, J. High Energy Phys. 01 (2008) 019.

[17] L. Vecchi, Multitrace deformations, gamow states, and stability of AdS/CFT, J. High Energy Phys. 04 (2011) 056.

[18] J. Erdmenger, C. Hoyos, A. O'Bannon, and J. Wu, A holographic model of the kondo effect, J. High Energy Phys. 12 (2013) 086.

[19] S. A. Hartnoll, C. P. Herzog, and G. T. Horowitz, Holographic superconductors, J. High Energy Phys. 12 (2008) 015.

[20] M. Headrick, S. Kitchen, and T. Wiseman, A new approach to static numerical relativity and its application to kaluzaklein black holes, Classical Quantum Gravity 27, 035002 (2010).

[21] P. Figueras, J. Lucietti, and T. Wiseman, Ricci solitons, ricci flow and strongly coupled CFT in the schwarzschild unruh or boulware vacua, Classical Quantum Gravity 28, 215018 (2011).

[22] P. O. Fedichev, A. E. Muryshev, and G. V. Shlyapnikov, Dissipative dynamics of a kink state in a bose-condensed gas, Phys. Rev. A 60, 3220 (1999).

[23] A. Cetoli, J. Brand, R. G. Scott, F. Dalfovo, and L. P. Pitaevskii, Snake instability of dark solitons in fermionic superfluids, Phys. Rev. A 88, 043639 (2013).

[24] B. P. Anderson, P. C. Haljan, C. A. Regal, D. L. Feder, L. A. Collins, C. W. Clark, and E. A. Cornell, Watching Dark Solitons Decay into Vortex Rings in a Bose-Einstein Condensate, Phys. Rev. Lett. 86, 2926 (2001).
[25] Z. Dutton, Observation of quantum shock waves created with ultra- compressed slow light pulses in a bose-einstein condensate, Science 293, 663 (2001).

[26] T. Yefsah, A. T. Sommer, M. J. H. Ku, L. W. Cheuk, W. Ji, W. S. Bakr, and M.W. Zwierlein, Heavy solitons in a fermionic superfluid, Nature (London) 499, 426 (2013).

[27] A. E. Muryshev, H. B. van Linden van den Heuvell, and G. V. Shlyapnikov, Stability of standing matter waves in a trap, Phys. Rev. A 60, R2665 (1999).

[28] T. Busch and J. R. Anglin, Motion of Dark Solitons in Trapped Bose-Einstein Condensates, Phys. Rev. Lett. 84, 2298 (2000).

[29] T. Busch and J. R. Anglin, Dark-Bright Solitons in Inhomogeneous Bose-Einstein Condensates, Phys. Rev. Lett. 87, 010401 (2001).

[30] D. M. Gangardt and A. Kamenev, Quantum Decay of Dark Solitons, Phys. Rev. Lett. 104, 190402 (2010).

[31] M. Guo, E. Keski-Vakkuri, H. Liu, Y. Tian, and H. Zhang, Dynamical phase transition from nonequilibrium dynamics of dark solitons, Phys. Rev. Lett. 124, 031601 (2020).

[32] S. Cremonini, L. Li, and J. Ren, Intertwined orders in holography: pair and charge density waves, J. High Energy Phys. 08 (2017) 081.

[33] Ó. J. C. Dias, J. E. Santos, and B. Way, Numerical methods for finding stationary gravitational solutions, Classical Quantum Gravity 33, 133001 (2016).

[34] S. Lan, W. Liu, and Y. Tian, Static structures of the BCS-like holographic superfluid in AdS4 spacetime, Phys. Rev. D 95, 066013 (2017).

[35] T. Faulkner, G. T. Horowitz, and M. M. Roberts, Holographic quantum criticality from multi-trace deformations, J. High Energy Phys. 04 (2011) 051.

[36] C. Charmousis, B. Goutéraux, B. S. Kim, E. Kiritsis, and R. Meyer, Effective holographic theories for low-temperature condensed matter systems, J. High Energy Phys. 11 (2010) 151.

[37] Ó. J. C. Dias, G. T. Horowitz, N. Iqbal, and J. E. Santos, Vortices in holographic superfluids and superconductors as conformal defects, J. High Energy Phys. 04 (2014) 096.

[38] G. Tallarita, R. Auzzi, and A. Peterson, The holographic non-abelian vortex, J. High Energy Phys. 03 (2019) 114.

[39] V. Balasubramanian and P. Kraus, A stress tensor for anti-de sitter gravity, Commun. Math. Phys. 208, 413 (1999).

[40] K. Skenderis, Asymptotically anti-de sitter spacetimes and their stress energy tensor, Int. J. Mod. Phys. A 16, 740 (2001).

[41] M. Guo, S. Lan, C. Niu, Y. Tian, and H. Zhang, Note on zero temperature holographic superfluids, Classical Quantum Gravity 33, 127001 (2016).

[42] Y.-Q. Wang and S. Liu, Holographic s-wave and p-wave josephson junction with backreaction, J. High Energy Phys. 11 (2016) 127. 